\title{
Concomitant Chemoradiation Using Gemcitabine in Locally Advanced Hepatocellular Carcinoma
}

\author{
Mohamed-Alaa-Eldeen H. Mohamed \\ Department of Clinical Oncology and Nuclear Medicine, Faculty of Medicine, Assiut University, Assiut, Egypt \\ Email: malaaassiut@gmail.com
}

How to cite this paper: Mohamed, M.-A.-E.H. (2018) Concomitant Chemoradiation Using Gemcitabine in Locally Advanced Hepatocellular Carcinoma. Journal of Cancer Therapy, 9, 1027-1038.

https://doi.org/10.4236/jct.2018.912084

Received: November 25, 2018

Accepted: December 26, 2018

Published: December 29, 2018

Copyright (C) 2018 by author and Scientific Research Publishing Inc. This work is licensed under the Creative Commons Attribution International License (CC BY 4.0).

http://creativecommons.org/licenses/by/4.0/

(c) (i) Open Access

\begin{abstract}
Purpose: Hepatocellular carcinoma (HCC) is one of the most common cancers worldwide. Most of patients present with advanced disease that cannot be treated with curative or ablative intent. The aim of the study was to assess the therapeutic benefit of concurrent chemoradiotherapy (CCRT) in patients with advanced HCC and to identify factors that predict OS. Methods: Patients with radiologically or biopsy proven primary HCC from 2010 to 2013 were included in the study. Gemcitabine was given in a dose of $70 \mathrm{mg} / \mathrm{m}^{2}$ once weekly for three weeks during external beam radiotherapy $30 \mathrm{~Gy} / 2$ Gy/fraction/five fractions per week in the Clinical Oncology Department, Assiut, Egypt. Results: Forty-nine patients were eligible but forty patients were evaluable for assessment. The partial response rate (PR) was 35\% (14 patients), 16 patients (40\%) had stable disease (SD) and the progressive disease was $25 \%$. Painful hepatomegaly was significantly relieved in $70 \%$ of patients. Transaminases rose as grade 3 toxicity in $15 \%$ of patients. Longer overall survival (OS) was significantly observed in patients with performance status 0 , child-Pugh A, Barcelona Clinic Liver cancer Stage B, PR and $\alpha$ fetoprotein level decrement. Conclusion: Concurrent chemoradiotherapt provides an acceptable response and palliation of pain in advances HCC. Our findings showed that better survival for patients with good performance and liver functions.
\end{abstract}

\section{Keywords}

Chemoradiation, Gemcitabine, Hapatocellular Carcinoma, Survival

\section{Introduction}

Hepatocellular carcinoma (HCC) accounting for 75\% - 85\% of primary liver 
cancer [1]. High risk patients should enter into a surveillance program to diagnose the disease in early stage and increase survival [2].

Primary liver cancer is the sixth most commonly occurring cancer in the world and the fourth cause of cancer mortality. The highest incidence of HCC is in Eastern and South-Eastern Asia. Egypt, Gambia and Guninea represent the highest incidence in Northern and western Africa [3].

In Egypt, liver cancer represents $23.8 \%$ of cancer incidence in both sexes. The proportions and age standardized incidence rate (ASR) of liver cancer were highest in Lower Egypt (29.6\%), less in Middle, and least in Upper Egypt (8.2\%) [4].

Risk factors for the incidence of HCC include chronic infection of hepatitis B, hepatitis $\mathrm{C}$, alcoholic hepatitis, non-alcoholic fatty liver disease and liver cirrhosis from any cause [5].

Staging of patients with HCC is important both for the prognostication and deciding the treatment. In this tumor, the prognosis depends upon the tumor stage and the liver function impairment due to underlying cirrhosis liver [6].

Many staging systems have been used for staging of HCC taking in to account the tumor status (known by tumor size and number, presence of vascular invasion and extrahepatic spread), liver function (defined by the Child-Pugh's class which determines the levels of serum bilirubin, albumin, presence of ascites and portal hypertension) and general health status of the patient (graded by the Eastern Cooperative Oncology Group classification) [7].

Staging systems commonly used in HCC include the European systems \{Barcelona Clinic Liver Cancer (BCLC) staging system [8]\} and Asian systems \{Okuda staging system [9]\}.

HCC mostly remain within the liver, but multifocality and vascular invasion are common [10]. For treatment selection, an acceptable balance between surviving the remaining liver function and tumor control is a challenge [11].

Surgical resection of HCC is indicated for limited cases of HCCs. Radiofrequency ablation is used for the treatment of HCCs of certain size and number [12]. Transcatheter arterial chemoembolization (TACE)) using iodized poppy seed oil, Lipiodol and anticancer drugs are used for the treatment of unresectable HCC [13].

Currently, the Korean Liver Cancer Study Group and National Comprehensive Cancer Network guidelines recommend combined radiotherapy (RT) and chemotherapy for selected cases of HCC. Concurrent chemoradiotherapy can augment the therapeutic successes for locally advanced HCC [14].

The aim of the study was to evaluate the efficacy of concurrent gemcitabine and external beam radiotherapy in locally advanced hepatocellular carcinoma and to define prognostic factors for survival among patients.

\section{Patients and Methods}

This prospective single arm phase II study was conducted in Clinical Oncology 
department, Faculty of Medicine, Assiut University, Egypt to assess the efficacy and safety profile of concurrent gemcitabine and external beam radiotherapy for the treatment of unresectable hepatocelluar carcinoma (HCC).

\section{Inclusion criteria}

Patients were included if they had a pathological confirmation of HCC or had fulfilled the American Association for the Study of Liver Diseases (AASLD) [15] criteria of the appearance of nodules on single dynamic technique either computed tomography (CT) or magnetic resonance imaging (MRI) showing intense arterial uptake followed by "washout" of contrast in the venous-delayed phases.

Patients were included if they have stage B/C using Barcelona Clinic Liver cancer (BCLC) staging system [16]. Patients were not candidate for transarterial embolization.

\section{Exclusion criteria}

Patients were excluded if they had Easter cooperative oncology group (ECOG) performance status $>2$, extrhepatic metastases, a history of radiotherapy to the abdominal area or concurrent chemoradiotherapy was not the first treatment.

A baseline evaluation included medical history, clinical examination including signs of liver cirrhosis (ascites and encephalopathy), performance status scoring, laboratory studies including complete blood count, liver and renal function tests, scoring of liver functions by Child-Pugh scoring, serologic markers for hepatitis $\mathrm{B}$ or $\mathrm{C}$ viruses and $\alpha$-fetoprotein level (reference range, 0 to $15 \mathrm{ng} / \mathrm{mL}$ ). Imaging studies (dynamic CT abdomen or MRI of the liver) was required. Bone scan or brain magnetic resonance was done when indicated. The Child-Pugh score was calculated from obtained clinical and laboratory data.

\section{Treatment plan}

Gemcitabine was given as IV infusion over 30 minutes in a dose of $70 \mathrm{mg} / \mathrm{m}^{2}$ once weekly for three weeks started with day 1 of radiotherapy.

Gross tumor volume (GTV) defined radiological as abnormal area seen on CT scan and clinical target volume (CTV) defined as GTV plus $1 \mathrm{~cm}$ margin. We irradiated the planning tumor volume as CTV plus $0.5-2.5 \mathrm{~cm}$ (determined under fluoroscopy) in the cranial-caudal dimensions to account for liver motion from respiration. A 2-dimensional radiotherapy using anteroposterior and posteroanterior fields was used. The radiation treatment was delivered with ${ }^{60} \mathrm{Co}$ up to $30 \mathrm{~Gy}$. Radiation therapy was administered once daily, five fractions a week at $2 \mathrm{~Gy} /$ fraction.

\section{Evaluation of response and toxicity}

Assessment of response was characterized according to the modified-Response Evaluation Criteria in Solid Tumors (m-RECIST) [17] defined as: Complete response, partial response, stable disease and progressive disease.

Evaluation of the disease and monitoring of toxicity was done monthly by physical/laboratory examination and contrast enhanced imaging CT/MRI scan every 3 - 6 months for 2 years then annually.

In patients in whom the serum AFP level was elevated before treatment, assay was done one month after termination of treatment, every 3 months for 2 years, 
then 6 monthly. Patients with chronic intermittent hepatic pain were assessed according to pain scale of 0 to 10 (patient self assessment). Patients with underlying liver disease were closely observed, monitored and appropriately treated.

Toxic effects were assessed weekly during chemoradiation and after completion of chemoradiotherapy by using common toxicity criteria of the National Cancer Institute version 4.0 [18].

\section{Statistical analysis}

The outcome measurement of this treatment included response rate, progression-free survival and overall survival.

Progression-free survival (PFS) was calculated from the date of initiation of treatment to the documented date of progression or date of last follow-up.

Overall survival (OS) is defined as the time from the start of chemoradiation to the date of death or last follow-up.

Baseline characteristics of the patients were expressed as percentage and median. Survival analysis was estimated with the use of Kaplan-Meier method. Differences in survival among their prognostic variables were tested by using log-rank test. A P value $<0.05$ was considered statistically significant. All statistical analysis was performed using SPSS 21.0 (SPSS, Inc. Chicago, IL).

\section{Results}

Between July 1, 2010 and June 30, 2013, 49 patients with HCC were included in the study; of these, 9 patients did not complete the treatment plan and the remaining 40 patients were included in the analyses. The baseline patient characteristics are listed in Table 1.

Seventy-five percent of the evaluable patients were men and $83 \%$ of the cohort had liver cirrhosis. Thirty (75\%) patients had HCC associated with hepatitis C infection and Alpha-Fetoprotein was elevated in $85 \%$ of patients. Regarding staging of HCC patients in the current study, twenty patients (65\%) had Child-Pugh stage B and sixteen (40\%) patients had BCLC stage C. In addition, thirty patients (75\%) had painful hepatomegaly.

Forty patients were evaluable for response assessment of concurrent chemoradiation. According to $\mathrm{m}$-RECIST for evaluation of response, fourteen patients (35\%) had a partial radiological tumor response, sixteen patients (40\%) had stable disease and ten patients (25\%) had progressive disease. Among patients who had painful hepatomegaly, the response rate to chemoradiation was significantly different $(70 \%$ vs. $30 \%, P=0.03)$. The median overall survival was 7 months (95\% confidence interval [CI] 2.63 - 11.36) and the progression-free survival was 4 months (95\% CI, 2.51 - 5.48). Treatment outcome is summarized in Table 2.

Prognostic factors for overall survival identified by univariate analysis were performance status 0, Alpha-fetoprotein decrement, BCLC stag B, Child-Pugh stag $\mathrm{A}$ and partial response were significantly associated with survival as reported in Table 3.

On performing multivariate analysis using all prognostic factors, ECOG 
Table 1. Clinical characteristics of patients with locally advanced hepatocellular carcinoma.

\begin{tabular}{|c|c|c|}
\hline Characteristics & Patients No. $=49(\%)$ & Evaluable patients $\mathrm{N}=\mathbf{4 0}(\%)$ \\
\hline Median age "range, years" & $60 “ 43-70 ”$ & 58 “43 - 70” \\
\hline \multicolumn{3}{|l|}{ Sex: } \\
\hline - Male & $38(77.55)$ & $30(75)$ \\
\hline - Female & $11(22.45)$ & $10(25)$ \\
\hline \multicolumn{3}{|l|}{ Performance status "ECOG": } \\
\hline - 0 & $15(30.61)$ & $14(35)$ \\
\hline - 1 & $19(38.78)$ & $18(45)$ \\
\hline - 2 & $15(30.61)$ & $8(20)$ \\
\hline \multicolumn{3}{|l|}{ Cirrhosis: } \\
\hline - Yes & $42(85.71)$ & $33(82.5)$ \\
\hline - No & $7(14.29)$ & $7(17.5)$ \\
\hline \multicolumn{3}{|l|}{ Etiology: } \\
\hline - Hepatitis B virus & $7(14.29)$ & $5(12.5)$ \\
\hline - Hepatitis $\mathrm{C}$ virus & $36(73.47$ & $30(75)$ \\
\hline - Hepatitis B \& C virus & $3(6.12)$ & $2(5)$ \\
\hline - Unknown & $3(6.12)$ & $3(7.5)$ \\
\hline \multicolumn{3}{|l|}{ Portal vein thromboses: } \\
\hline - Yes & $29(59.18)$ & $20(50)$ \\
\hline - No & $20(40.82)$ & $20(50)$ \\
\hline \multicolumn{3}{|l|}{ Alpha-Fetoprotein: } \\
\hline - Elevated & $43(87.8 \%)$ & $34(85)$ \\
\hline - Normal & $6(12.2 \%)$ & $6(15)$ \\
\hline \multicolumn{3}{|c|}{ Liver function by Child-Pugh stage: } \\
\hline - $\mathrm{A}$ & $11(22.45)$ & $9(22.5)$ \\
\hline - $\mathrm{B}$ & $29(59.18)$ & $26(65)$ \\
\hline - $\mathrm{C}$ & $9(18.37)$ & $5(12.5)$ \\
\hline \multicolumn{3}{|l|}{ BCLC stage*: } \\
\hline - $\mathrm{B}$ & $17(34.69)$ & $16(40)$ \\
\hline - $\mathrm{C}$ & $32(65.31)$ & $24(60)$ \\
\hline \multicolumn{3}{|l|}{ Pain (painful hepatomegaly): } \\
\hline - Yes & $30(61.22))$ & $30(75))$ \\
\hline - No & $19(38.78)$ & $10(25)$ \\
\hline
\end{tabular}

Note: $\mathrm{ECOG}=$ Eastern Cooperative Oncology Group performance status, ${ }^{*}$ Barcelona Clinic Liver Cancer.

performance status ( 0 vs. 1 vs. 2 ) and Child-Pugh stage (A vs. B \& C) were independent prognostic factors for progression-free survival (Table 4). 
Table 2. Treatment outcomes after concomitant gemcitabine and radiotherapy in patients with advanced hepatocellular carcinoma.

\begin{tabular}{cc}
\hline Outcomes & No. $=\mathbf{4 9}(\%)$ \\
\hline Partial response & $14(35)$ \\
Stable disease & $16(40)$ \\
Progressive disease & $10(25)$ \\
Not evaluable & 9 \\
Painful hepatomegaly: & \\
Responders (21/30) & $21(70)$ \\
Non-responders (9/30) & $9(30)$ \\
P value & 0.03 \\
Median overall survival, 95\% CI & $7.0(2.63-11.36)$ \\
Median progression-free survival, 95\% CI & $4.0(2.51-5.48)$ \\
\hline
\end{tabular}

Note: $\mathrm{CI}=$ confidence interval.

Table 3. Univariate analysis of prognostic factors for progression-free survival (PFS) after concomitant gemcitabine and radiotherapy in patients with hepatocellular carcinoma.

\begin{tabular}{|c|c|c|}
\hline Factor & Median PFS (95\% CI) & p-value \\
\hline \multicolumn{3}{|c|}{ Performance status ECOG: } \\
\hline - 0 & $7(5.46-8.53)$ & \multirow{3}{*}{$0.001^{*}$} \\
\hline - 1 & $4(3.31-4.68)$ & \\
\hline - 2 & $2(1.23-2.74)$ & \\
\hline \multicolumn{3}{|c|}{ Alpha-Fetoprotein decrement: } \\
\hline - Yes & $7(6.24-7.75)$ & \multirow[b]{2}{*}{$0.001^{\star}$} \\
\hline - No & $4(2.88-5.11)$ & \\
\hline \multicolumn{3}{|c|}{ BCLC staging system: } \\
\hline - $\mathrm{B}$ & $7(5.94-8.05)$ & \multirow[b]{2}{*}{$0.001^{*}$} \\
\hline - $\mathrm{C}$ & $3(2.85-4.02)$ & \\
\hline \multicolumn{3}{|c|}{ Liver function by Child-Pugh stage: } \\
\hline - $\mathrm{A}$ & $6(3.49-8.50)$ & \multirow{3}{*}{$0.001^{*}$} \\
\hline - $\mathrm{B}$ & $4(3.35-4.65)$ & \\
\hline - $\mathrm{C}$ & $2(2.00-2.00)$ & \\
\hline \multicolumn{3}{|c|}{ Response: } \\
\hline - $\quad \mathrm{PR}$ & $7(5.79-8.21)$ & \multirow{3}{*}{$0.001^{*}$} \\
\hline - $\mathrm{SD}$ & $4(3.51-4.48)$ & \\
\hline - $\mathrm{PD}$ & $2(2.00-2.00)$ & \\
\hline
\end{tabular}

Note: $\mathrm{ECOG}=$ Eastern Cooperative oncology Group, $\mathrm{BCLC}=$ Barcelona Clinic Liver Cancer, $\mathrm{PR}=$ partial response, $\mathrm{SD}=$ stable disease, $\mathrm{PD}$ progressive disease. $\mathrm{CI}=$ confidence interval, ${ }^{*}=$ significant values. 
Table 4. Multivariate analysis of prognostic factors of progression-free survival of the study cohort of patients with hepatocellular carcinoma treated with chemoradiation.

\begin{tabular}{|c|c|c|c|}
\hline Factors & $\beta$ & SE & Sig $(P)$ \\
\hline \multicolumn{4}{|l|}{ - Performance status } \\
\hline (0 vs. 1 vs. 2 ) & 0.574 & 0.044 & $0.001^{\star}$ \\
\hline \multicolumn{4}{|l|}{ - AFP decrement } \\
\hline (Yes vs. No) & 0.159 & 0.763 & 0.231 \\
\hline \multicolumn{4}{|l|}{$-\quad$ BCLC } \\
\hline (B vs. C) & 0.026 & 0.665 & 0.866 \\
\hline \multicolumn{4}{|l|}{ - Child-Pugh stage } \\
\hline (A vs. B vs. C) & 0.353 & 1.523 & $0.001^{*}$ \\
\hline \multicolumn{4}{|l|}{ - Response } \\
\hline (PR vs. $\mathrm{SD}$ vs. $\mathrm{PD}$ ) & 0.178 & 0.331 & 0.205 \\
\hline
\end{tabular}

Note: $\mathrm{AFP}=$ Alpha-fetoprotein, $\mathrm{BCLC}=$ Barcelona Clinic Liver Cancer, $\mathrm{PR}=$ partial response, $\mathrm{SD}=$ stable disease, $\mathrm{PD}$ progressive disease, ${ }^{*}=$ significant values, $\beta$ : regression coefficient, $\mathrm{SE}$ : standard error, Sig $(\mathrm{P})$ : probability value

Twenty percent of patients had grade II diarrhea and rise in serum tranaminase level while fifteen percent of patients had grade III rise in serum transaminase level. Grading of toxicity of chemoradiation is shown in Table 5.

Partial responders of HCC patients treated by concurrent chemoradiation had significantly longer survival than patients showing stable disease and progressive disease (median OS 7 months for PR vs. 4 months for SD and 2 months for PD). Kaplan-Meier curve of overall survival of patients according to the types of response is presented in Figure 1.

Median overall survival of 40 HCC patients treated by concurrent chemoradiation was 7 months. Kaplan-Meier curve of overall survival of patients with HCC included in the analysis is presented in Figure 2.

\section{Discussion}

Hepatocellular carcinoma is an important health issue, particularly in regions where viral hepatitis prevalence is high. In Egypt, Africa HCC represents 23.8\% of cancer incidence [4]. The current study investigates the clinical outcomes of concurrent external beam radiotherapy and weekly gemcitabine in patients with HCC.

In this study, the partial response (PR) rate to concurrent chemradiation (CCRT) was 35\% was achieved. These results were comparable to the results of the study done by Masood AI et al. [19] who reported $36 \%$ as overall response rate.

Grade 3 toxicity of CCRT in the current study was seen in $15 \%$ of patients as raised liver enzymes. Toxicity of CCRT using systemic chemotherapy had acceptable toxicity in locally advanced HCC [19]. 


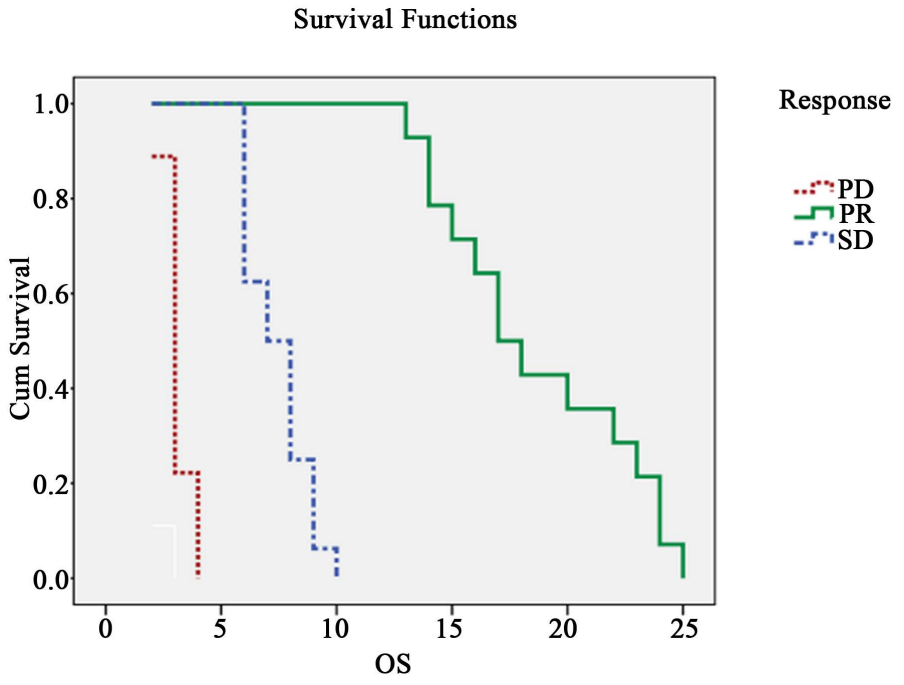

Figure 1. Overall survival (OS) of hepatocellular carcinoma patients treated with chemoradiation according to response, PR: partial responders [median OS 7 months, 95\% confidence interval (CI) 5.79 - 8.21], SD: stable disease [median OS 4 months, 95\%CI 3.51 4.48], and PD: progressive disease [median OS 2 months, 95\% CI 2.0 - 2.0]. Significant longer OS of partial responders $(\mathrm{p}=0.001)$.

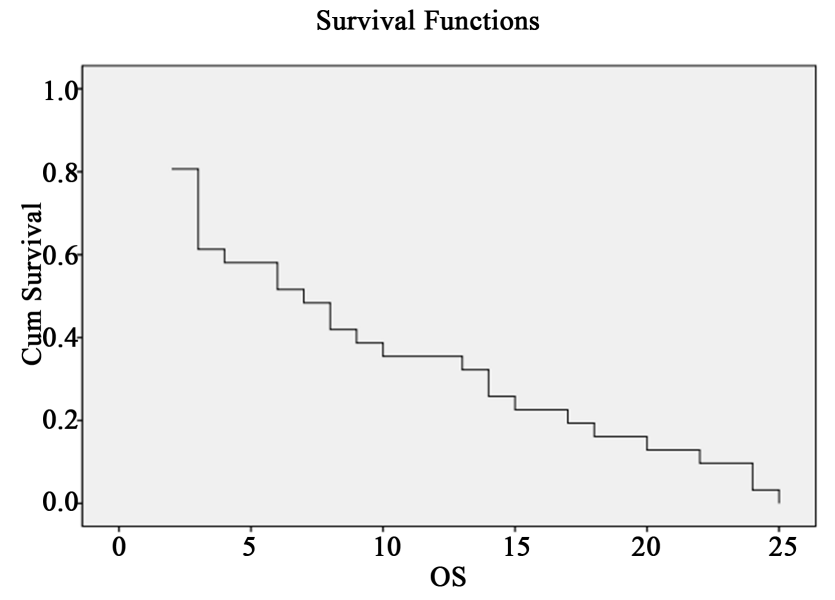

Figure 2. Overall survival (OS) of hepatocellular carcinoma patients treated with chemoradiation (median OS = 7 months, 95\% confidence interval; 2.63 - 11.36).

Table 5. Toxicity of radiotherapy and weekly gemcitabine in the treatment of patients with locally advanced hepatocellular carcinoma.

\begin{tabular}{|c|c|c|}
\hline \multicolumn{2}{|c|}{ Toxicity (NCICTC, v. 4) } & \multirow{2}{*}{$\begin{array}{c}\text { No. of patients }=40 \\
6(15 \%)\end{array}$} \\
\hline & - Vomiting & \\
\hline \multirow[t]{3}{*}{ Grade II } & - Diarrhea & $8(20 \%)$ \\
\hline & - Transaminase & $8(20 \%)$ \\
\hline & - Vomiting & $4(10 \%)$ \\
\hline \multirow[t]{2}{*}{ Grade III } & - Diarrhea & $4(10 \%)$ \\
\hline & - Transaminase & $6(15 \%)$ \\
\hline
\end{tabular}

Note: NCICTC = National Cancer Institute Common Terminology Criteria for Adverse Events, version 4. 
The combination of radiotherapy (RT) and locoregional therapy such as transarterial chemoemblization (TACE) has become one of the effective therapies for HCC. Radiotherapy can be used as a complement to incomplete TACE and a treatment for liver thrombus [20].

A meta-analysis was done to evaluate the effect of TACE plus RT versus TACE alone on tumor response, survival, and adverse events. Patients receiving TACE plus RT showed significantly better complete response and 1-year survival compared with TACE alone and the survival benefit increased for 5-year survival [21].

Another study reported that patients with unresectable HCC treated with RT, either as CCRT or in combination with TACE, showed effective intrahepatic control and prolonged survival [22].

Update results of a study on patients with advanced HCC who had portal vein thrombosis revealed that survival was significantly longer in patients treated with hepatic arterial infusion of chemotherapy + RT than with sorafenib monotherapy [23].

Safe delivery of ablative doses of RT to focal liver tumors is limited by the tolerance of the liver and adjacent tissues. 3-diminsional conformal radiotherapy (3DCRT) and intensity modulated radiotherapy (IMRT) are techniques used to target tumors with higher doses while sparing normal surrounding tissues. Image-guided RT (IGRT) is currently used to assist in the delivery of 3DRT, IMRT and sterotactic RT along with respiratory motion compensation and tumor visualization [24].

In the present study radiotherapy was prescribed for delivering palliative and not curative treatment. Radiation dose was limited to 30 Gy to prevent radiotherapy-induces liver disease (RILD) if the whole liver included in the target volume because the mean liver dose associated with a 5\% risk of RILD is 28 Gy at 2 Gy per fraction for primary liver cancer [25].

In the analyses of prognostic factors, the CCRT treatment in this study showed a significantly higher OS with performance status 0 , BCLC stage B, Child-Pugh A, partial responders and AFP responders.

Other prognostic factors of OS in patients with HCC with CP-A versus B was reported by Hung $\mathrm{HH}$ et al. which are non-hepatitis $\mathrm{C}$ virus carrier, serum albumin $\leq 4 \mathrm{~g} / \mathrm{dL}$, aspartate aminotransferase $>45 \mathrm{U} / \mathrm{L}, \alpha$-fetoprotein $>20 \mathrm{ng} / \mathrm{mL}$, tumor size $>3 \mathrm{~cm}$, multinodularity, vascular invasion, and noncurative therapies resulted in worse survival but not the Child-Pugh numeric score [26].

In patients with huge HCC $(>10 \mathrm{~cm})$ treated by multimodality including RT, the significant prognostic factors of survival were number of tumors, initial AFP level, percent AFP decrement, treatment response, and hepatic resection [27].

The correlation between AFP response and survival outcome in patients with HCC has been reported in other studies as a prognostic factor for survival [28] [29].

Regarding the role of radiotherapy in palliation of pain, the present study showed that a significant number of patients had a symptomatic relief of pain 
(painful hepatomegaly). These results are in agreement with the results of the study done by Hammad AY et al., who reported that RT was the best palliative therapy for HCC and provided a survival benefit for patients with stage IV disease. In their study, $18 \%$ of patients received RT to the liver as palliative treatment of pain [30].

This study has some of the limitations of a prospective study. First are the less restrictive inclusion criteria for acceptance of participants, lack of follow-up data about tumor progression was it inside or outside the radiation field and whether there was reactivation of viral hepatitis after liver irradiation. Second are the limitations in administration of advanced radiation technologies with the delivery of higher external beam radiation dose and combining it with novel target/immunotherapy agents.

\section{Conclusion}

Concurrent chemoradiotherapt provides an acceptable response with minimal toxicity in advances HCC. Our findings showed that better survival for patients with a good performance and liver functions. CCRT will be recommended for patients with advanced HCC for palliation of pain.

\section{Acknowledgements}

The author acknowledges patients who accept to participate in this study, colleagues and employee of the department for their assessment in data collection and analysis.

\section{Ethical Approval}

All procedures performed in the study which involve participants were in accordance with the ethical standard of the Faculty of Medicine, Assiut University, Egypt and the national research ethics committee and have been performed in accordance with the ethical standards as laid down in 1964 Declaration of Helsinki and its later amendments or comparable ethical standards.

This protocol was approved by the Ethics Committee of Faculty of Medicine, Assuit University, Egypt. A written informed consent was obtained before inclusion in the study.

\section{Conflicts of Interest}

The author declares that I have no conflict of interest.

\section{References}

[1] London, W.T., Petrick, J.L. and McGlynn, K.A. (2018) Liver Cancer. In: Thun, M.J., Linet, M.S., Cerhan, J.R., Haiman, C.A., Schottenfeld D., Eds., Cancer Epidemiology and Prevention, 4th Edition, Oxford University Press, New York, 635-660.

[2] Lersritwimanmaen, P. and Nimanong, S. (2018) Hepatocellular Carcinoma Surveillance: Benefit of Serum Alfa-Fetoprotein in Real-World Practice. Euroasian Journal of Hepato-Gastroenterology, 8, 83-87. 
[3] Bray, F., Ferlay, J., Soerjomataram, I., Siegel, R.L., Torre, L.A. and Jemal, A. (2018) Global Cancer Statistics: GLOBOCAN Estimates of Incidence and Mortality Worldwide for 36 Cancers in 185 Countries. CA: A Cancer Journal for Clinicians, 68, 394-424. https://doi.org/10.3322/caac.21492

[4] Ibrahim, A.S., Khaled, H.M., Mikhail, N.H., Baraka, H. and Kamel, H. (2014) Cancer Incidence in Egypt: Results of the National Population-Based Cancer Registry Program. Journal of Cancer Epidemiology, Article ID: 437971. https://doi.org/10.1155/2014/437971

[5] Mittal, S. and El-Serag, H.B. (2013) Epidemiology of Hepatocellular Carcinoma: Consider the Population. Journal of Clinical Gastroenterology, 47, S2-S6.

[6] Bruix, J. and Llovet, J.M. (2002) Prognostic Prediction and Treatment Strategy in Hepatocellular Carcinoma. Hepatology, 35, 519-524.

https://doi.org/10.1053/jhep.2002.32089

[7] Duseja, A. (2014) Staging of Hepatocellular Carcinoma. Journal of Clinical and EXperimental Hepatology, 4, S74-S79. https://doi.org/10.1016/j.jceh.2014.03.045

[8] Llovet, J.M., Bru, C. and Bruix, J. (1999) Prognosis of Hepatocellular Carcinoma: The BCLC Staging Classification. Seminars in Liver Disease, 19, 329-338. https://doi.org/10.1055/s-2007-1007122

[9] Okuda, K., Ohtsuki, T., Obata, H., Tomimatsu, M., Okazaki, N., Hasegawa, H., Nakajima, Y. and Ohnishi, K. (1985) Natural History of Hepatocellular Carcinoma and Prognosis in Relation to Treatment. Cancer, 56, 918-928. https://doi.org/10.1002/1097-0142(19850815)56:4<918::AID-CNCR2820560437>3.0 .CO;2-E

[10] Herrero, J.I., Sangro, B., Quiroga, J., Pardo, F., Herraiz, M., Cienfuegos, J.A. and Prieto J. (2001) Influence of Tumor Characteristics on the Outcome of Liver Transplantation among Patients with Liver Cirrhosis and Hepatocellular Carcinoma. Liver Transplantation, 7, 631-636. https://doi.org/10.1053/jlts.2001.25458

[11] Llovet, J.M. (2005) Updated Treatment Approach to Hepatocellular Carcinoma. Journal of Gastroenterology, 40, 225-235. https://doi.org/10.1007/s00535-005-1566-3

[12] Wang, Y., Luo, Q., Li, Y., Deng, S., Wei, S. and Li, X. (2014) Radiofrequency Ablation versus Hepatic Resection for Small Hepatocellular Carcinomas: A Meta-Analysis of Randomized and Nonrandomized Controlled Trials. PLoS One, 9, e84484. https://doi.org/10.1371/journal.pone.0084484

[13] Yamashita, T. and Kaneko, S. (2013) Treatment Strategies for Hepatocellular Carcinoma in Japan. Hepatology Research, 43, 44-50. https://doi.org/10.1111/j.1872-034X.2012.01029.x

[14] Yoon, H.I. and Seong, J. (2014) Multimodality Treatment Involving Radiotherapy for Advanced Liver-Confined Hepatocellular Carcinoma. Oncology, 87, 90-98. https://doi.org/10.1159/000368151

[15] Bruix, J. and Sherman, M. (2010) American Association for the Study of Liver Diseases (AASLD) Practical Guidelines. Management of Hepatocellular Carcinoma: An Update. Hepatology, 1-36.

[16] Forner, A., Reig, M.E., de Lope, C.R. and Bruix, J. (2010) Current Strategy for Staging and Treatment: The BCLC Update and Future Prospects. Seminars in Liver Disease, 30, 61-74. https://doi.org/10.1055/s-0030-1247133

[17] Lencioni, R. and Llovet, J.M. (2010) Modified RECIST (mRECIST) Assessment for Hepatocellular Carcinoma. Seminars in Liver Disease, 30, 52-60.

https://doi.org/10.1055/s-0030-1247132 
[18] (2010) Common Toxicity Criteria Classification Version 4.03. http://ctep.info.nih,gov/reporting/ctc.html

[19] Masood, A.I., Javed, A.A., Mateen, A. and Gurchani, S.A. (2009) Concomitant Chemoradiation Using Gemcitabine in Locally Advanced Hepatocellular Carcinoma. The Chinese-German Journal of Clinical Oncology, 11, 130-133.

[20] Kondo, Y., Kimura, O. and Shimosegawa, T. (2015) Radiation Therapy Has Been Shown to Be Adaptable for Various Stages of Hepatocellular Carcinoma. World Journal of Gastroenterology, 21, 94-101.

[21] Huo, Y.R. and Eslick, G.D. (2015) Transcatheter Arterial Chemoembolization plus Radiotherapy Compared with Chemoembolization Alone for Hepatocellular Carcinoma: A Systematic Review and Meta-Analysis. JAMA Oncology, 1, 756-765. https://doi.org/10.1001/jamaoncol.2015.2189

[22] Kim, K.H., Kim, M.S. and Chang, J.S. (2014) Therapeutic Benefit of Radiotherapy in Huge $(\geq 10 \mathrm{~cm})$ Unresectable Hepatocellular Carcinoma. Liver International, 34, 784-794. https://doi.org/10.1111/liv.12436

[23] Kodama, K., Kawaoka, T., Aikata, H., Kobayashi, T., Uchikawa, S., Ohya, K., Kodama, K., et al. (2018) Comparison of Outcome of Hepatic Arterial Infusion Chemotherapy Combined with Radiotherapy and Sorafenib for Advanced Hepatocellular Carcinoma Patients with Major Portal Vein Tumor Thrombosis. Oncology, 94, 215-222. https://doi.org/10.1159/000486483

[24] Klein, J. and Dawson, L.A. (2013) Hepatocellular Carcinoma Radiation Therapy: Review of Evidence and Future Opportunities. International Journal of Radiation Oncology, Biology, Physics, 87, 22-32. https://doi.org/10.1016/j.ijrobp.2012.08.043

[25] Dawson, L.A. and Ten Haken, R.K. (2005) Partial Volume Tolerance to Liver Radiation. Seminars in Radiation Oncology, 15, 279-283. https://doi.org/10.1016/j.semradonc.2005.04.005

[26] Hung, H.H., Chao, Y., Chiou, Y.Y., Li, C.P., Lee, R.C., Huo, T.I., et al. (2014) A Comparison of Clinical Manifestations and Prognoses between Patients with Hepatocellular Carcinoma and Child-Pugh Scores of 5 or 6. Medicine (Baltimore), 93, e348. https://doi.org/10.1097/MD.0000000000000348

[27] Han, H.J., Kim, M.S., Cha, J., Choi, J.S., Han, K.H. and Seong, J. (2014) Multimodality Treatment with Radiotherapy for Huge Hepatocellular Carcinoma. Oncology, 87, 82-89. https://doi.org/10.1159/000368150

[28] Lombardi, G., Pastorelli, D., Zustovich, F., Farina, L., Furini, A., Cappetta, V., et al. (2010) Factors Predicting Time-to-Progression (TTP) and Overall Survival (OS) in Patients with Unresectable Hepatocellular Carcinoma (uHCC) Treated by Combination Gemcitabine (G) and Pegylated Liposomal Doxorubicin (PLD) Chemotherapy. Journal of Clinical Oncology, 28, e14618-e14618.

[29] Kim, B.K., Ahn, S.H., Seong, J.S., Park, J.Y., Kim, D.Y., Kim, J.K., et al. (2011) Early $\alpha$-Fetoprotein Response as a Predictor for Clinical Outcome after Localized Concurrent Chemoradiotherapy for Advanced Hepatocellular Carcinoma. Liver International, 31, 369-376. https://doi.org/10.1111/j.1478-3231.2010.02368.x

[30] Hammad, A.Y., Robbins, J.R., Turaga, K.K., Christians, K.K., Gamblin, T.C. and Johnston, F.M. (2017) Palliative Interventions for Hepatocellular Carcinoma Patients: Analysis of the National Cancer Database. Annals of Palliative Medicine, 6, 26-35. https://doi.org/10.21037/apm.2016.11.02 